\title{
A DUAL CHARACTERISATION OF THE RADON-NIKODYM PROPERTY
}

\author{
M. BACHIR AND A. DANILLIDIS
}

\begin{abstract}
We prove that a Banach space $X$ has the Radon-Nikodym property if, and only if, every weak*-lower semicontinuous convex continuous function $f$ of $X^{*}$ is Gâteaux differentiable at some point of its domain with derivative in the predual space $X$.
\end{abstract}

\section{INTRODUCTION}

Collier [5] has shown that a Banach space $X$ has the Radon-Nikodym property if, and only if, all weak*-lower semicontinuous convex continuous functions on the dual space $X^{*}$ are generically Fréchet differentiable. (Such a dual space was called in [5] weak*Asplund.) In this article we give the following characterisation of the Radon-Nikodym property in terms of Gâteaux derivatives.

THEOREM 1. A Banach space $X$ has the Radon-Nikodym property if, and only if, every weak*-lower semicontinuous convex continuous function on $X^{*}$ is Gâteaux differentiable at some point of its domain with derivative in the predual space $X$.

Since Fréchet derivatives of weak*-lower semicontinuous convex continuous functions of $X^{*}$ are always elements of $X$ (see [7], for example), the improvement upon the aforementioned result of Collier consists on replacing the Fréchet derivative by Gâteaux and on passing from a dense differentiability assumption to the existence of the derivative at one point.

If $X$ does not have the Radon-Nikodym property, then it is possible to have nowhere Fréchet differentiable weak*-lower semicontinuous convex continuous functions on $X^{*}$ for which the set of points where the Gâteaux derivative exists and belongs to the predual space is dense (see Proposition 8). Concurrently, it is also possible to have weak*lower semicontinuous convex continuous functions on $X^{*}$ that are generically Gâteaux differentiable with all derivatives in $X^{* *} \backslash X$. Indeed, consider the Banach space $X=$ $c_{0}(\mathbb{N})$, its dual space $X^{*}=\ell^{1}(\mathbb{N})$ and the function $g(x)=\|x\|_{1}$, see [10, Example $\left.1.4(\mathrm{~b})\right]$ for details.

Received 6th January, 2000

The authors wish to thank R. Deville, G. Godefroy and N. Hadjisavvas for useful discussions. The research of the second author was supported by the TMR post-doctoral grant ERBFMBI CT 983381.

Copyright Clearance Centre, Inc. Serial-fee code: 0004-9727/00 $\$ A 2.00+0.00$. 
Let us finally note that characterisations of the Radon-Nikodym property for dual Banach spaces in terms of the Gâteaux derivative are recently established by Giles in [8, Theorem 2].

The proof of Theorem 1 is given in Section 3, while in the following section we fix our notation and we recall relevant definitions.

\section{Preliminaries}

In the sequel, $(X,\|\|$.$) will be a Banach space and \left(X^{*},\|\|.\right)$ will be its dual. We denote by $B_{X}$ the closed unit ball of $X$ and by $\mathbb{R}$ (respectively, $\mathbb{N}$ ) the set of all real (respectively, positive integer) numbers. For any $x \in X$ and any $p \in X^{*}$ we denote by $\langle p, x\rangle$ the value of the functional $p$ at the point $x$. Similarly, for any $z^{* *}$ in $X^{* *}$ we denote by $\left\langle p, z^{* *}\right\rangle$ the value of $z^{* *}$ at $p$. We also denote by $\overline{c o} F$ the closed convex hull of the set $F$. For any non-empty closed bounded subset $F$ of $X$ we denote by $\psi_{F}$ the indicator function of $F\left(\psi_{F}(x):=0\right.$, if $x \in F$ and $+\infty$, if $\left.x \notin F\right)$ and by $\psi_{F}^{*}$ its Fenchel conjugate, that is, for all $p \in X^{*}$

$$
\psi_{F}^{*}(p)=\sup _{x \in F}\langle p, x\rangle
$$

It is known that $\psi_{F}^{*}$ is a weak*-lower semicontinuous convex continuous function. (The latter follows from the fact that the boundedness of $F$ yields dom $\psi_{F}^{*}=X^{*}$.) We also recall that every weak ${ }^{*}$-lower semicontinuous convex continuous function $g: X^{*} \rightarrow \mathbb{R} \cup$ $\{+\infty\}$ coincides with the first conjugate $f^{*}$ of a lower semicontinuous convex function $f$ defined on $X$ (take $f:=g^{*}$ ). We denote by $\operatorname{dom} g:=\left\{p \in X^{*}: g(p)<+\infty\right\}$ the domain of the function $g$. Then, the Fenchel-Moreau subdifferential $\partial g$ of $g$ at any $p_{0} \in$ dom $g$ is defined as follows:

$$
\partial g\left(p_{0}\right)=\left\{z^{* *} \in X^{* *}: g(p)-g\left(p_{0}\right) \geqslant\left\langle p-p_{0}, z^{* *}\right\rangle, \forall p \in X^{*}\right\}
$$

If $p_{0} \in X^{*} \backslash$ dom $g$, then we set $\partial g\left(p_{0}\right)=\emptyset$.

Given a closed subset $F$ of $X$ and a point $x_{0}$ of $F$ we say that $x_{0}$ is a strongly exposed point of $F$, if there exists $p_{0} \in X^{*}$ such that any sequence $\left\{x_{n}\right\}_{n \geqslant 1}$ in $F$ satisfying $\lim _{n \rightarrow+\infty}\left\langle p_{0}, x_{n}\right\rangle=\sup _{x \in F}\left\langle p_{0}, x\right\rangle$ converges to $x_{0}$ in the norm topology. In such case we say that the functional $p_{0}$ strongly exposes $x_{0}$ in $F$. We denote by se $(F)$ the set of strongly exposed points of $F$.

We now introduce the notion of a weakly exposed point, which will be useful in the sequel, see Lemma 5.

DEFINITION 2. Let $F$ be a closed subset of $X$. A point $x_{0} \in F$ is called a weakly exposed point in $F$, if there exists $p_{0} \in X^{*}$ such that any sequence $\left\{x_{n}\right\}_{n \geqslant 1}$ in $F$ with $\lim _{n \rightarrow+\infty}\left\langle p_{0}, x_{n}\right\rangle=\sup _{x \in F}\left\langle p_{0}, x\right\rangle$ weakly converges to $x_{0}$. 
In the case of the above definition we say that the functional $p_{0}$ weakly exposes $x_{0}$ in $F$. It follows easily that $p_{0}$ attains its unique maximum on $F$ at $x_{0}$, hence in particular $x_{0}$ is an extreme point of $F$. We denote by we $(F)$ the set of weakly exposed points of $F$. Furthermore, a point $x_{0}$ is called a point of continuity of $F$, if the identity mapping id : $\left(F, \Im_{w}\right) \rightarrow\left(F, \Im_{\|\cdot\| \|}\right)$ is continuous, where $\Im_{w}$ (respectively, $\left.\Im_{\|\cdot\| \|}\right)$ denotes the relative weak (respectively, norm) topology of $F$. It follows directly that $x_{0}$ is a strongly exposed point of $F$ if, and only if, it is both weakly exposed and a point of continuity of $F$. Finally, a point $x_{0}$ is called weakly denting (or strongly extreme, according to the terminology in $\left[4\right.$, p.67]), if for any relatively weakly open subset $W$ in $F$ containing $x_{0}$ there exist $p \in X^{*}$ and $\alpha>0$ such that the set $\left\{x \in F:\langle p, x\rangle>\left\langle p, x_{0}\right\rangle-\alpha\right\}$ is included in $W$.

\section{Proof of the MaIN RESUlt}

The proof of Theorem 1 is based on the following result of Bourgain [3, Chapter 1; Theorem 4]. (For a proof in English, see [4, Corollary 3.7.6].)

THEOREM 3. A Banach space $X$ has the Radon-Nikodym property if, and only if, every nonempty closed convex bounded subset $F$ of $X$ has at least one weakly denting point.

We can easily deduce the following corollary. The analogous result for dual Banach spaces is given in [8, Theorem 4].

COROLlary 4. For a Banach space $X$, the following are equivalent:

(i) $X$ has the Radon-Nikodym property

(ii) Every closed convex bounded subset of $X$ is the closed convex hull of its weakly exposed points.

(iii) Every nonempty closed convex bounded subset of $X$ has at least one weakly exposed point.

Proof: It is known ([4, Corollary 3.5.7], [10, Theorem 5.21], for example) that a Banach space $X$ has the Radon-Nikodym property if, and only if, every closed convex bounded subset of $X$ is the closed convex hull of its strongly exposed points. This shows that (i) $\Longrightarrow$ (ii). Implication (ii) $\Longrightarrow$ (iii) is trivial, while (iii) $\Longrightarrow$ (i) follows from Theorem 3 and the observation that every weakly exposed point of $F$ is weakly denting.

REMARK 1. A weakly denting point is not in general weakly exposed, even in finite dimensions. Indeed, let $X=\mathbb{R}^{2}, F=\left\{\left(x_{1}, x_{2}\right): f\left(x_{1}\right) \leqslant x_{2} \leqslant g\left(x_{1}\right)\right\}$, where $f(x)=$ $\max \left\{0, x^{3}\right\}$ and $g(x)=x+1$, and $\bar{x}=(0,0)$. Then $\bar{x}$ is a weakly denting point of the compact convex set $F$, without being weakly exposed.

REMARK 2. A Banach space $X$ has the Radon-Nikodym property if, and only if, for every nonempty closed convex bounded subset $F$ of $X$ we have se $(F) \neq \emptyset$. However, if $X$ does not have the Radon-Nikodym property, then the fact that we $(F) \neq \emptyset$ (or even 
that $\overline{c o}($ we $(F))=F$ ) for some closed convex bounded subset $F$ of $X$ does not necessarily imply that $\operatorname{se}(F) \neq \emptyset$. (Consider the subset $F$ of $c_{0}(\mathbb{N})$ defined by (12) in Proposition 8 and Claims 1 and 2 therein.)

We shall finally need the following lemma.

LEMMA 5. Let $X$ be a Banach space and $F$ be any non-empty closed convex bounded subset $X$. Then the following are equivalent:

(i) The function $\psi_{F}^{*}$ is Gâteaux differentiable at $p_{0}$ with derivative $x_{0} \in X$.

(ii) $x_{0} \in F$ and the functional $p_{0}$ is weakly exposing $x_{0}$ in $F$.

Proof: (i) $\Longrightarrow$ (ii): Assume that (i) holds. Since $x_{0}=\nabla^{G} \psi_{F}^{*}\left(p_{0}\right)$ (where $\nabla^{G} \psi_{F}^{*}$ denotes the Gâteaux derivative of $\psi_{F}^{*}$ ), we obviously have $x_{0} \in \partial \psi_{F}^{*}\left(p_{0}\right)$, that is for all $p \in X^{*}$

$$
\psi_{F}^{*}(p)-\psi_{F}^{*}\left(p_{0}\right) \geqslant\left\langle p-p_{0}, x_{0}\right\rangle
$$

For $p=0$ we obtain

$$
\psi_{F}^{*}\left(p_{0}\right):=\sup _{x \in F}\left\langle p_{0}, x\right\rangle=\left\langle p_{0}, x_{0}\right\rangle
$$

Let now $\left\{x_{n}\right\}_{n \geqslant 1}$ be a sequence in $F$ such that

$$
\lim _{n \rightarrow+\infty}\left\langle p_{0}, x_{n}\right\rangle=\sup _{x \in F}\left\langle p_{0}, x\right\rangle .
$$

It suffices to show that $\left\{x_{n}\right\}_{n \geqslant 1}$ weakly converges to $x_{0}$. (Then, since the weak and the norm closure of the convex set $F$ coincide, it will also follow that $x_{0} \in F$.)

Let us assume, towards a contradiction, that there exists a subsequence $\left\{x_{n_{k}}\right\}_{k \geqslant 1}$ of $\left\{x_{n}\right\}_{n \geqslant 1}, h \in X^{*}$ and $\alpha>0$ such that for all $k \geqslant 1$

$$
\left\langle h, x_{n_{k}}\right\rangle-\left\langle h, x_{0}\right\rangle>\alpha
$$

Thanks to (3) and (4), we can consider $\varepsilon_{n} \searrow 0^{+}$in a way that

$$
\left\langle p_{0}, x_{n}\right\rangle \geqslant\left\langle p_{0}, x_{0}\right\rangle-\varepsilon_{n}
$$

Since $\psi_{F}^{*}(p) \geqslant\left\langle p, x_{n}\right\rangle$, using (3) we get

$$
\psi_{F}^{*}(p) \geqslant \psi_{F}^{*}\left(p_{0}\right)+\left\langle p, x_{n}\right\rangle-\left\langle p_{0}, x_{0}\right\rangle,
$$

which in view of $(6)$ yields

$$
\psi_{F}^{*}(p) \geqslant \psi_{F}^{*}\left(p_{0}\right)+\left\langle p-p_{0}, x_{n}\right\rangle-\varepsilon_{n}
$$

Set $t_{n}=2 \varepsilon_{n} / \alpha$. Then for $p=p_{0}+t_{n} h$ relation (7) yields

$$
\left(\psi_{F}^{*}\right)\left(p_{0}+t_{n} h\right)-\left(\psi_{F}^{*}\right)\left(p_{0}\right) \geqslant\left\langle t_{n} h, x_{n}\right\rangle-\varepsilon_{n}
$$


for all $n \geqslant 1$. In view of $(5)$ this implies

$$
\frac{\left(\psi_{F}^{*}\right)\left(p_{0}+t_{n_{k}} h\right)-\left(\psi_{F}^{*}\right)\left(p_{0}\right)}{t_{n_{k}}}-\left\langle h, x_{0}\right\rangle \geqslant \frac{\alpha}{2}>0
$$

for all $k \geqslant 1$. It follows that $x_{0}$ is not the Gâteaux derivative of $\psi_{F}^{*}$ at $p_{0}$, hence a contradiction.

(ii) $\rightarrow$ (i): Suppose that $p_{0}$ is weakly exposing $x_{0}$ in $F$, hence in particular $\left\langle p_{0}, x_{0}\right\rangle=$ $\sup _{x \in F}\left\langle p_{0}, x\right\rangle$. It follows easily from (2) that $x_{0} \in \partial \psi_{F}^{*}\left(p_{0}\right)$. Let us now suppose that (i) does not hold. Then there exist $\varepsilon>0, h \in X^{*}$ with $\|h\| \leqslant 1$ and $t_{n} \searrow 0^{+}$such that

$$
\left(\psi_{F}^{*}\right)\left(p_{0}+t_{n} h\right)-\left(\psi_{F}^{*}\right)\left(p_{0}\right)>\left\langle t_{n} h, x_{0}\right\rangle+\varepsilon t_{n} .
$$

For every $n \geqslant 1$, choose $x_{n}$ in $F$ such that

$$
\left\langle p_{0}+t_{n} h, x_{n}\right\rangle>\left(\psi_{F}^{*}\right)\left(p_{0}+t_{n} h\right)-\frac{t_{n}}{n}
$$

Since $\left(\psi_{F}^{*}\right)\left(p_{0}\right) \geqslant\left\langle p_{0}, x_{n}\right\rangle$, the above inequality yields

$$
\left\langle p_{0}+t_{n} h, x_{n}\right\rangle-\left\langle p_{0}, x_{n}\right\rangle>\left(\psi_{F}^{*}\right)\left(p_{0}+t_{n} h\right)-\left(\psi_{F}^{*}\right)\left(p_{0}\right)-\frac{t_{n}}{n} .
$$

Hence

$$
\left\langle t_{n} h, x_{n}\right\rangle>\left(\psi_{F}^{*}\right)\left(p_{0}+t_{n} h\right)-\left(\psi_{F}^{*}\right)\left(p_{0}\right)-\frac{t_{n}}{n} .
$$

Combining (8) and (10) we conclude

$$
\left\langle h, x_{n}-x_{0}\right\rangle>\varepsilon-\frac{1}{n}
$$

which shows that $\left\{x_{n}\right\}_{n \geqslant 1}$ does not weakly converge to $x_{0}$. However, since the sequence $\left\{x_{n}\right\}_{n \geqslant 1}$ is bounded and the function $\psi_{F}^{*}$ is continuous, relation (9) yields $\lim _{n \rightarrow+\infty}\left\langle p_{0}, x_{n}\right\rangle=$ $\left(\psi_{F}^{*}\right)\left(p_{0}\right)$, obtaining thus a contradiction to Definition 2 .

REMARK. The above proof was inspired from techniques developed in [2] where a connection between well-posed problems and differentiability was established. Results in the same spirit are also established in [6, Section 1], via a different approach. We are grateful to $C$. Zălinescu for bringing the aforementioned reference to our attention.

Proof of Theorem 1: The "only if" part follows from the result of Collier [5] and the fact that the Fréchet derivatives of weak*-lower semicontinuous convex continuous functions on $X^{*}$ always belong to the predual space $X$ (see [7], for example).

For the "if" part, let $F$ be any closed convex bounded subset of $X$. Then the function $\psi_{F}^{*}$ of $X^{*}$ (given in (1)) is weak*-lower semicontinuous convex and continuous. From our hypothesis and Lemma 5 we conclude that we $(F) \neq \emptyset$. Since $F$ is arbitrary, Corollary 4 asserts that $X$ has the Radon-Nikodym property. 
Let us recall that a Banach space $X$ is called weakly sequentially complete, if every weakly Cauchy sequence of $X$ is weakly converging in $X$. A typical example of a nonreflexive weakly sequentially complete Banach space is the space $L^{1}(\mu)$, where $\mu$ is a $\sigma$-finite positive measure. The following remark is due to Godefroy.

Corollary 6 . Let $X$ be a weakly sequentially complete Banach space. Then $X$ has the Radon-Nikodym property if, and only if, every weak*-lower semicontinuous convex continuous function on $X^{*}$ is Gâteaux differentiable at some point of its domain.

PROOF: The "only if" part is a direct consequence of Theorem 1. The "if" part follows from the following observation: if $F$ is a nonempty closed convex bounded subset of $X$, and if $\nabla^{G} \psi_{F}^{*}(p)$ is the Gâteaux derivative of the function $\psi_{F}^{*}$ at $p \in X^{*}$, then there exists $\left\{x_{n}\right\}_{n \geqslant 1}$ in $F$ that weakly*-converges to $\nabla^{G} \psi_{F}^{*}(p)$ (see the proof of Lemma 5 (i) $\Longrightarrow\left(\right.$ ii)). It follows that $\left\{x_{n}\right\}_{n \geqslant 1}$ is a weakly Cauchy sequence, hence in view of our hypothesis $\nabla^{G} \psi_{F}^{*}(p) \in X$. (For similar considerations, see also [9].) We conclude by Lemma 5 (i) $\Longrightarrow$ (ii) and Corollary 4 (iii) $\Longrightarrow($ i).

Lemma 5 has also the following consequence. (The proof below is similar to [10, Theorem 5.20].)

Corollary 7. Let $F$ be a closed convex bounded subset of $X$. If $\psi_{F}^{*}$ is Gâteaux differentiable in a dense subset of $X^{*}$ with derivatives in $X$, then $F=\overline{c o}($ we $(F)$ ).

Proof: Since $F$ is bounded, we have $\operatorname{dom}\left(\psi_{F}^{*}\right)=X^{*}$. (In particular the function $\psi_{F}^{*}$ is convex and Lipschitz.) Since $F$ is closed and convex, we have $\overline{c o}$ (we $\left.(F)\right) \subseteq F$. Let us suppose, towards a contradiction, that there exists some $x_{0}$ in $F \backslash \overline{\text { co }}$ (we $(F)$ ). Then by applying the Hahn-Banach theorem, we can find $p \in X^{*}(p \neq 0)$ and $\alpha \in \mathbb{R}$ such that

$$
\sup \{\langle p, x\rangle: x \in \overline{\mathrm{co}}(\text { we }(F))\}<\alpha<\left\langle p, x_{0}\right\rangle \text {. }
$$

Set $D=\left\{q \in X^{*}: \exists \nabla^{G}\left(\psi_{F}^{*}\right)(q) \in X\right\}$. Since $D$ is dense in $X^{*}$, we can find $q \in D$ close to $p$ such that $z:=\nabla^{G}\left(\psi_{F}^{*}\right)(q) \in X$ and

$$
\sup \{\langle q, x\rangle: x \in \overline{\mathrm{co}}(\text { we }(F))\}<\alpha<\left\langle q, x_{0}\right\rangle \text {. }
$$

By Lemma 5 we conclude that $z \in$ we $(F)$ and that the functional $q$ weakly exposes $z$. This clearly contradicts (11).

The space $c_{0}(\mathbb{N})$ is a typical example of a Banach space without the Radon-Nikodym property. In this case, as already mentioned in Section 1 , the norm $\|\cdot\|_{1}$ provides an example of a weak*-lower semicontinuous convex continuous function of $\ell^{1}(\mathbb{N})$, which is generically Gâteaux differentiable with all derivatives in $X^{* *} \backslash X$. In the following proposition we give an example of a (nowhere Fréchet differentiable) weak*-lower semicontinuous convex continuous function of $\ell^{1}(\mathbb{N})$, which is Gâteaux differentiable with derivatives in the predual space in a dense set. 
Proposition 8. Let $X=c_{0}(\mathbb{N})$. Then there exists a weak ${ }^{*}$-lower semicontinuous convex continuous function $f: X^{*} \rightarrow \mathbb{R}$ such that:

(i) there exists a dense subset $D$ of $X^{*}$ such that $f$ is Gâteaux differentiable at every point of $D$ with derivative in the predual space ;

(ii) $f$ is nowhere Fréchet differentiable.

Proof: Set $X=c_{0}(\mathbb{N})$ and consider the set

$$
F=B_{X} \cap c_{0}^{+}(\mathbb{N}):=\left\{x=\left(x^{i}\right)_{i}:\|x\|_{\infty} \leqslant 1 \text { and } x^{i} \geqslant 0(\forall i \in \mathbb{N})\right\} .
$$

It is easily seen that $F$ is closed convex bounded and that

$$
\operatorname{ext}(F)=\left\{x \in F: x^{i} \in\{0,1\} \text { for all } i\right\}
$$

where ext $(F)$ denotes the set of the extreme points of $F$.

ClaIM 1. Let $\bar{x} \in \operatorname{ext}(F)$ and consider the finite set

$$
I_{\bar{x}}=\left\{i \in \mathbb{N}: \bar{x}^{i}=1\right\} .
$$

Then any functional $p=\left(p^{i}\right)_{i}$ of $X^{*}:=\ell^{1}(\mathbb{N})$ satisfying

$$
\begin{array}{ll}
p^{i}>0, & \text { if } i \in I_{\bar{x}} \\
p^{i}<0, & \text { if } i \in \mathbb{N} \backslash I_{\bar{x}}
\end{array}
$$

weakly exposes the point $\bar{x}$. In particular ext $(F)=$ we $(F)$ (and hence we $(F) \neq \emptyset$ ).

Proof of Claim 1: Let $\bar{x} \in \operatorname{ext}(F), I_{\bar{x}}=\left\{i \in \mathbb{N}: \bar{x}^{i}=1\right\}$ and consider any $p$ in $\ell^{1}(N)$ satisfying (14). We first note that for all $x \in F$ and all $i \in \mathbb{N}$ we have

$$
p^{i} x^{i} \leqslant p^{i} \bar{x}^{i}
$$

It follows that $\langle p, x\rangle \leqslant\langle p, \bar{x}\rangle$, for all $x \in F$, that is, $\langle p, \bar{x}\rangle=\sup _{x \in F}\langle p, x\rangle$. Take now any sequence $\left\{x_{n}\right\}_{n \geqslant 1}$ in $F$ such that $\lim _{n \rightarrow+\infty}\left\langle p, x_{n}\right\rangle=\langle p, \bar{x}\rangle$. We show that

$$
\lim _{n \rightarrow+\infty} x_{n}^{i}=\bar{x}^{i}
$$

for all $i \geqslant 0$. Indeed, assume that for some $i_{0}(16)$ does not hold. Then there exist a subsequence $\left\{x_{n_{k}}^{i_{0}}\right\}_{k \geqslant 1}$ of $\left\{x_{n}^{i_{0}}\right\}_{n \geqslant 1}, \varepsilon>0$ and $k_{0} \in \mathbb{N}$ such that for all $k \geqslant k_{0}$

$$
\left|x_{n_{k}}^{i_{0}}-\bar{x}^{i_{0}}\right|>\frac{\varepsilon}{\left|p^{i_{0}}\right|} \text {. }
$$

Using (15) we infer that

$$
p^{i_{0}} x_{n_{k}}^{i_{0}}<p^{i_{0}} \bar{x}^{i_{0}}-\varepsilon
$$


Combining with (15) we get $\left\langle p, x_{n_{k}}\right\rangle\left\langle\langle p, \bar{x}\rangle-\varepsilon\right.$, for all $k \geqslant k_{0}$. This contradicts the fact that $\left\langle p, x_{n}\right\rangle \rightarrow\langle p, \bar{x}\rangle$. It follows that (16) holds for all $i \geqslant 0$. Since the sequence $\left\{x_{n}\right\}_{n \geqslant 1}$ is bounded, we conclude from (16) that $x_{n} \stackrel{w}{\longrightarrow} \bar{x}$. Hence the functional $p$ is weakly exposing $\bar{x}$ in $F$. Since every weakly exposed point is obviously extreme, the proof of the claim is complete.

Claim 2: $\operatorname{se}(F)=\emptyset$.

Proof of Claim 2: It clearly suffices to show that any point $\bar{x}$ in we $(F)$ is not a point of continuity for $F$. To this end, take any $\bar{x} \in$ we $(F)$ and consider the sequence $\left\{x_{n}\right\}_{n \geqslant 1}$ in $F$ with

$$
x_{n}^{i}= \begin{cases}1 & \text { if } i \in I_{\bar{x}} \cup\{n\} \\ 0 & \text { elsewhere }\end{cases}
$$

where $I_{\bar{x}}$ is given by (13). Then it follows easily that $x_{n} \stackrel{w}{\rightarrow} \bar{x}$. On the other hand, for $n$ sufficiently large, we have $\left\|x_{n}-\bar{x}\right\|_{\infty}=1$.

Consider now the weak*-lower semicontinuous convex continuous function $\psi_{F}^{*}$ : $\ell^{1}(\mathbb{N}) \rightarrow \mathbb{R}$ defined by

$$
\psi_{F}^{*}(p):=\sup _{x \in F}\langle p, x\rangle=\left\|p_{+}\right\|_{1}
$$

where $\|\cdot\|_{1}$ is the usual norm of $\ell^{1}(\mathbb{N})$ and

$$
p_{+}^{i}= \begin{cases}p^{i} & \text { if } p^{i}>0 \\ 0 & \text { if } p^{i} \leqslant 0\end{cases}
$$

Let us denote by $D$ the set of all functionals $p=\left(p^{i}\right)_{i}$ in $\ell^{1}(\mathbb{N})$ satisfying (14) for some finite (possibly empty) subset $I$ of $\mathbb{N}$. For every such functional $p$, consider the point $\bar{x}=\left(\bar{x}^{i}\right)_{i}$ of $c_{0}(\mathbb{N})$ defined by

$$
\bar{x}^{i}= \begin{cases}1 & \text { if } i \in I \\ 0 & \text { if } i \in \mathbb{N} \backslash I .\end{cases}
$$

Then $\bar{x} \in F$ and $I=I_{\bar{x}}$ (where $I_{\bar{x}}$ is given in (13)). It follows by Claim 1 that the functional $p$ weakly exposes $\bar{x}$. Applying Lemma 5 (ii) $\rightarrow$ (i) we conclude that $\bar{x}$ is the Gâteaux derivative of $\psi_{F}^{*}$ at $p$.

We now show that $D$ is dense in $\ell^{1}(\mathbb{N})$. Indeed, take any $q=\left(q^{i}\right)_{i}$ in $\ell^{1}(\mathbb{N})$ and any $\varepsilon>0$. Then for some $n_{0} \in \mathbb{N}$ we have:

$$
\|q\|_{1} \leqslant \sum_{i=0}^{n_{0}}\left|q^{i}\right|+\frac{\varepsilon}{2} .
$$

Consider now the functional $p=\left(p^{i}\right)_{i}$ defined by

$$
p^{i}=\left\{\begin{array}{cl}
q^{i} & \text { if } i \leqslant n_{0} \text { and } q^{i} \neq 0 \\
-\frac{\varepsilon}{2^{i+2}} & \text { elsewhere. }
\end{array}\right.
$$


It is easily seen that $p \in D$. Moreover,

$$
\|q-p\|_{1}=\sum_{i=0}^{+\infty}\left|q^{i}-p^{i}\right| \leqslant \sum_{i>n_{0}}^{+\infty}\left|q^{i}\right|+\sum_{i=0}^{+\infty} \frac{\varepsilon}{2^{i+2}} \leqslant \varepsilon .
$$

We have shown that the function $\psi_{F}^{*}$ is densely Gâteaux differentiable with derivatives in the predual space $X$. On the other hand, since by Claim 2 the set $F$ has no strongly exposed points, it follows from $[1$, p. 450$]$ that $\psi_{F}^{*}$ is nowhere Fréchet differentiable.

Let us finally note that the function $\psi_{F}^{*}$ is in fact generically Gâteaux differentiable. Indeed, it is easily seen that for every $p=\left(p^{i}\right)_{i}$ with $p^{i} \neq 0$ for all $i$, we have $\nabla^{G} \psi_{F}^{*}(p)=$ $z^{* *}$ where $z^{* *} \in \ell^{\infty}(\mathbb{N})$ is given by

$$
\left(z^{* *}\right)^{i}= \begin{cases}1 & \text { if } p^{i}>0 \\ 0 & \text { if } p^{i}<0\end{cases}
$$

\section{REFERENCES}

[1] E. Asplund and R.T. Rockafellar, 'Gradients of convex functions', Trans. Amer. Math. Soc. 139 (1969), 433-467.

[2] M. Bachir, 'On generic differentiability and Banach-Stone's theorem', (preprint 37p, University of Bordeaux, 1999), C. R. Acad. Sci. Paris 330 (2000), 687-690.

[3] J. Bourgain, 'La propriété de Radon-Nikodym', Publ. Math. Univ. Pierre et Marie Curie 36 (1979).

[4] R. Bourgin, Geometric aspects of convex sets with the Radon-Nikodym property, Lecture Notes in Mathematics 993 (Springer-Verlag, Berlin, Heidelberg, New York, 1983).

[5] J. Collier, 'The dual of a space with the Radon-Nikodym property', Pacific J. Math. 64 (1976), 103-106.

[6] A. Dontchev and T. Zolezzi, Well-posed optimization problems, Lecture Notes in Mathematics 1543 (Springer-Verlag, Berlin, Heidelberg, New York, 1993).

[7] M. Fabian and V. Zizler, 'An elementary approach to some questions in higher order smoothness in Banach spaces', Extracta Math. (to appear).

[8] J. Giles, 'Comparable differentiability characterizations of two classes of Banach spaces', Bull. Austral. Math. Soc. 56 (1997), 263-272.

[9] G. Godefroy, 'Propriété de lissité de certaines normes', Math. Ann. 257 (1981), 185-189.

[10] R. Phelps, Convex functions, monotone operators and differentiability, Lecture Notes in Mathematics 1364, (2nd ed.) (Springer-Verlag, Berlin, Heidelberg, New York, 1993).

Laboratoire de Mathématiques Pures

Université Bordeaux I

351 cours de la Libération

33405 Talence Cedex

France

e-mail: bachir@math.u-bordeaux.fr
Laboratoire de Mathématiques Appliquées CNRS ERS 2055

Université de Pau et des Pays de l'Adour avenue de l'Université

$64000 \mathrm{Pau}$

France

e-mail: aris.daniilidis@univ-pau.fr 